\title{
Bases anatómicas para la disección quirúrgica de los cayados safenos 2. El confluente safenopoplíteo
}

\author{
JESÚS SÁNCHEZ \\ Hospinal Comaral de Jara (Huesara). España.
}

\begin{abstract}
RESUMEN
En el presence articulo presentamos la experiencia de nuestro Servicio en la disección del cayado de la vena salena externa en el transcurso de intervenciones por várices esenciales (47 cayados disecados en 41 paciences encre 1990 y 2001 ). junco con una amplia revisión biblionráfica acerca de la anatomía del confluente salenopoplíteo. Igualmente detallamos nuestros haIlazgos operarorios y se muesira la progresiva evolución que han experimentado las exploraciones preoperatorials y la récnica quirúrgica. También intencamos delinear la influencia que en la calidad de los diagnósticos anatómicos intraoperatorios han tenido la ecu-Doppler (1998) y la instauración de un prococolo operatorio estándar a partir del año 1996.
\end{abstract}

Palabras raves: Anaroma; diseccion: vena safena: lécnicas quirurgicas.

\section{ANATOMICAL BASIS TO SAFENA CAYADUS SUIRGICAL RESECTION PAR'T 2. THE SAPHENO-POPLITEAL ЛUNCTION \\ SUMMARY}

The purpose of this article is to show our experience on the dissection of short saphenous vein during surgical interventions due to essential varicose veins. Berween January 1990 and January 2001 rorty-seven saphencr-popliteal junctions were dissected in 41 patients. Moreover, we reviewed a broad bibliography regarding the anacomy of sapheno-popliceal junction. We explain both our operative findings and how important che preoperarive examination and surgical technique are. One relevant point is to outline the influence of echo-Doppler (uttrasound scan, 1998) and the implementarion of standard operative prococols in the gualicy of anatomical incraoperative diagnoses since 1996.

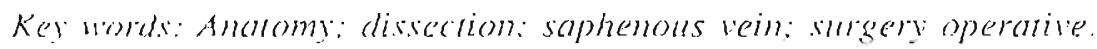

\section{INTRODUCCIÓN}

Todo cirujano dispuesto a praclicar una coreccla cirugía de las varices. ucbe posecr un conocimiento exhausrivo de la anatomia venosa de

\section{Correspandencia:}

Dr. Jesus Sánchez Beorlegui

Calle Migne!' Servet $196^{\circ} \mathrm{G}$

50002 Zaragoza. España

E-mail: hospinaliaca@iazfree.com las extremidades inleriores y de las variantes que pueden presentarsc. Esce dominio se hace particularmente necesario en el cayado de la venal saliena externa (VSE), zona en que la posusión de una léconica quirúrgica depurada es indispensable para cuitar complicaciones y prevenir recidivas varicosals. Las consecuencias médicolegales derívadias de la yarrogenial. de una recidiva precoz o de un mal resultado estético nunca pucden ser ignoradas por el cirujano 17cbológico ('). 
El tramo final de la VSE, al contrario que el de la safena interna (VSI), carece de una localización anacómica constante: puede encontrarse a alturas diferentes de la pierna o del muslo y tener como destino diversas venas del sistema venoso profundo (SVP). Incluso, en un número no despreciable de pacientes, puede faltar un cayado en su concepción "clásica" $\left({ }^{2,3}\right)$.

A la morfología variable de la terminación de la VSE, se suma la presencia de un sistema de perforantes e interconexiones con otros vasos no del todo conocidas $\left({ }^{4}\right)$, y la íntima proximidad de importantes elementos vásculo-nerviosos que circulan a través del hueco poplíteo. Todo ello hace que los resuitados de la cirugía en el territorio safeno externo sean mucho más aleatorios e impredecibles que en el interno $\left({ }^{5}\right)$, y la tasa de recidivas posquirúrgicas muy superior $\left(^{6}\right)$.

Las modernas técnicas de imagen, como el eco-Doppler color (EDC) y la llebografía selectiva, ofrecen al cirujano una ayuda esencial a la hora de buscar asesoramiento acerca del cayado de la VSE $\left({ }^{7-10}\right)$, pero no le eximen de poseer unos conocimientos anatómicos profundos.

Por ello, en el presente trabajo llevamos a cabo una amplia revisión bibliográfica acerca de la anatomía del confluente safenopoplítco y aportamos nuestra casuística. Igualmente, y aceptando el criterio expresado por múltiples autores $(2.5,9.11 .12)$, según el cual los buenos resultados quirúrgicos se asocian íntimamente a un diagnóstico anatómico intraoperatorio preciso y completo, intentamos establecer la influencia que han tenido el EDC (desdc 1998) y el establecimiento de un protocolo operatorio estándar (desde 1996) en la calidad de la cirugía en el territorio VSE en nuestro Scrvicio.

\section{Recucrdo anatómico de la vena safena externa}

La VSE drena la sangre de los rejidos subaponeurótico y de la piel situados en la parte posterior de la picrna y el muslo.
En su conliguración típica, el tronco venoso principal sc origina en el surco retromaleolar externo, a una distancia comprendida entre 3 y 8 centímetros por encima del relieve maleolar peroneal $\left({ }^{4.5} .11 .13\right)$. En dicho lugar se produce la unión de la vena marginal dorsal externa del pie y una rama venosa posterocentral o calcáned, que camina paralela al tendón de Aquiles. La conjunción de ambos vasos forma una imagen clásica de "Y" invertida. Esta morfología del origen safeno se reproduce en aproximadamente $80 \%$ de los casos $\left({ }^{9}\right)$.

La VSE camina hacia la línea media con una dirección oblicua y se sitúa en el surco intergemelar, en un plano subcutáneo por encima de la aponeurosis de la pierna. En un punto localizado en $60 \%$ de los casos en el tercio medio de la pierna ( 15 a 27 centímetros por encima del maléolo externo), el trayecto de la VSE se hace intraaponeurótico, ocupando un canal formado por un desdoblamiento de las hojas fasciales, en donde la acompañan tejido graso, una arteriola y ramos nerviosos íntimamente adheridos. En esta zona, el diámetro del tronco safeno alcanza una media de 4 milímetros (2 a $8 \mathrm{~mm}$ ).

En una localización variable, clásicamente al cruzar la interlínea articular de la rodilla, situada en el hueco poplíteo ( 3 a 5 centínetros por encima de la cabeza del peroné), la VSE se incurva, atraviesa la hoja profunda de la aponeurosis y camina a fundirse con la vena poplítea, a la que aborda por su cara posterior o postcrolateral (Figura 1), obscrvándose en prácticamente codos los casos una válvula ostial safenopoplítea. La profundidad de la unión, dependiendo del espesor del panículo adiposo, se sitúa en unos márgenes comprendidos entre 1,5 y 6 centínetros $\left({ }^{4.5 .9 .14}\right)$.

A partir de esta descripción "típica" del origen, trayecto y confluente de la VSE, registraremos los siguientes apuntes a partir de la literatura:

1. El modelo anteriormente descrito ("clásico") de la VSE se diseca en aproximadamente un $42 \%$ de los pacientes $\left({ }^{15}\right)$. En apenas $40 \%$ a 


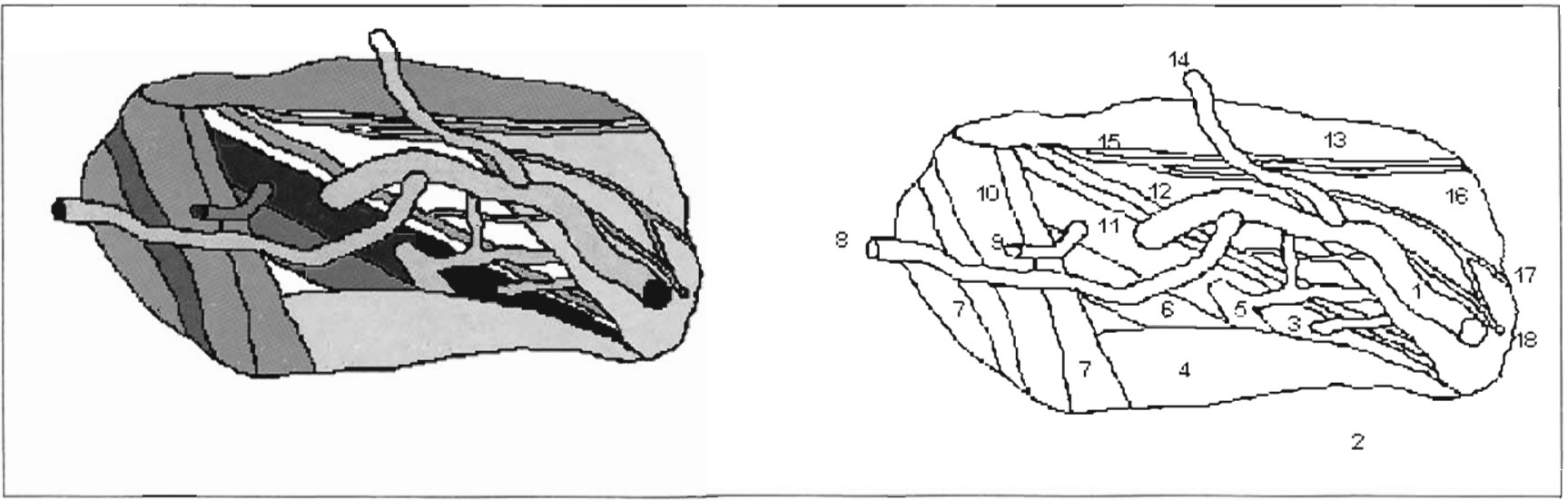

Figura 1.- Topografía general del bueco poplítco, incluyendo el tramo final de la VSE sigún la descripción "clásica". Aparecen representadas las dos colaterales más frecucntes, vena cabestrillo del inuslo y vena de Giacomini, y un cortocircuito de la VSE con las venas gemelares. 1) Vcna safena externa. 2) Fascia crural.

3) Vena del músculo soleo. 4) Músculo gastrocnemio, cabeza medial. 5) Venas gemclares, aquí lusionadas en "cañón de lusil". 6) Arteria poplítca. 7) Músculo semimembranoso. 8) Vena de Giacomini.

9) Vena del "área poplítea" o de Dodd. 10) Músculo semitendinoso. 11) Vcna poplítéa. 12) Nervio tibial.

13) Músculo bíceps femoral. 14) Vena cabestrillo del muslo. 15) Nervio peroneo común. 16) Músculo gastrocnemio, cabeza lateral. 17) Nervio cutánen sural lateral. 18) Nervio cutáneo sural medial.

$70 \%$ de los casos, es posible observar un cayado único y que vicrte todo su flujo en la vena poplítea $\left({ }^{16.17}\right)$.

2. En $90 \%$ de los cayados se puede disecar colaterales $\left({ }^{2}\right)$. Habitualmente, su número es interior a las de la VSI y, en algunos casos, pucden faltar por complcto, hecho éste excepcional en la safena magna $\left({ }^{18}\right)$.

3. Las principales variantes del cayado de la VSE pueden ser divididas en subgrupos, según el criterio clasificatorio cnipleado:

- Posición de la desembocadura: en 1926. Kosinski $\left({ }^{19}\right)$, basándose en disecciones en cadáveres, publicó unos porcentajes de $57,3 \%$ de cayados situados entre 0 y 5 centímetros por encima del plicgue poplítco y $33 \% 12$ o más centímetros por encima. Resumiendo múltiples reportes hasta nucstros días $(2,11.15,16.21) \cdot 24)$, estableceremos que la unión de la VSE con el sistema profundo es infrapoplítea en $1 \%$ a $10 \%$ de los casos, poplítca en $52 \%$ a $81 \%$ y suprapoplílea en $12 \%$ a $46 \%$ (Figura 2). No debe extrañar tanta variabilidad, scgún cl autor consultado, ya que toman diversas reforencias (todas cllas válidas por igual) y diferentes distancias desde las mismas, para establecer la altura de la unión.

Las uniones altas, de morfología similar a las de especies situadas por debajo del hombre en la escala filogenérica, se asocian con salicnas de mayor diámetro y no varicosas $\left({ }^{24}\right)$. Los confluentes localizados en el hueco poplítco o por debajo del mismo, son una adaptación cvolutiva asociadal a la marcha en bipedestación. En el ser humano, la VSE há expcrimentado una progresiva emigración cmbriológica en dirección caudal, junto con una marcada atrofia. Todo cllo provoca una disminución del calibre del vaso, reflujos desde el SVP y várices, excepcionales en los mamíferos cuadrúpedos $\left({ }^{24}\right)$.

- Vena en que se produce la desembocadura: poplícca o una de sus ramas (venas gemclares, vena del sóleo, venas de la rodilla), VSI, femoral superficial o femoral profunda $\left({ }^{11.25 .26}\right)$. En $46 \%$ de los pacienICs, una parte o la totalidad del tlujo VSE 


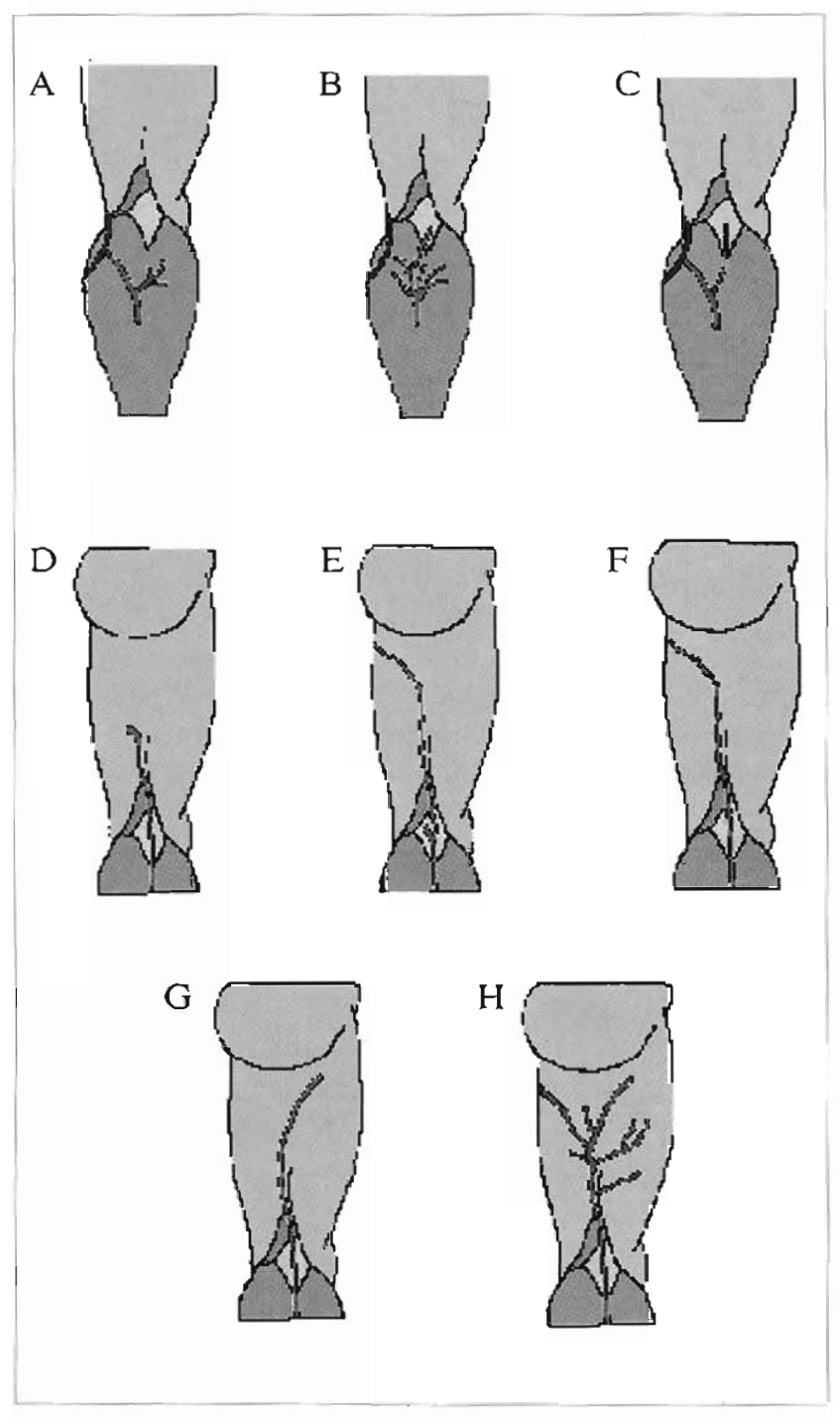

Figura 2.- Modalidades de terminación de la VSE. A. El tronco principal de la VSE, único o bifurcado, se vierte en la VSI y emite pequeñas ramas en el muslo que terminan en perforantes. B. No existe cayado; la VSE emite diversas ramas arborescentes quc se vierten en la VSI o en el sistema venoso profundo mediante perforantes. C. Similar al caso primero, pero un ramo secundario se une a la vena poplítea en el hueco, simulando un "cayddo" normal. D. Terminación alta, en el muslo, de la VSE. E. Cayado en posición clásica y coreccircuito con la VSI a traVés de una vena de Giacomini. F. Igual al anterior pero sin cayado ni conexión con la vena poplítea. G. La VSE se vierte en la femoral profunda a través de una perforante alta. H. No existe cayado, la VSE emite diversas ramas arborescentes cn el muslo. se vierte en la VSI $\left({ }^{15}\right)$. Para Moosman $\left({ }^{23}\right)$, $17,5 \%$ de las VSE no tiene algún tipo de conexión con la vena poplítea.

- Número de tributarias del cayado: Siendo la más constante la vena femoropoplítea, de Giacomini o de Hyrtl, que comunica los sistemas safenos externo e interno $\left({ }^{16,27}\right)$. Otras colaterales, directas o mediante cortocircuitos, pueden ser la vena cabestrillo del muslo o de Meyer, venas de los músculos gemelos, vena del sóleo y vena del área poplítea $(5.7 .12,13,20.28)$.

Las relaciones de la VSE con las venas gemelares son sumamente complejas, pudiendo adoptar diversas formas: terminaciones separadas, ostium común, tronco común, etc. $\left({ }^{3.7 .28 .29}\right)$ La fusión de ambas venas es más frecuente en safenas de implantación baja $\left.{ }^{8.16}\right)$.

La vena del área poplítea, de Dodd o vena "fosse poplitée" de Thiery, está presente en $40 \%$ de los pacientes. Cuando puedc ser observada, en $50 \%$ de los casos desemboca cn el cayado de la VSE, como una colateral del mismo $\left({ }^{5.20}\right)$.

4. Hasta $30 \%$ de los confluentes tiene una morfología totalmente atípica $\left({ }^{24}\right)$, por ejemplo (Figura 3):

- Anomalías del tronco principal de la VSE: safena desdoblada, presente hasta en $6 \%$ de los casos $\left({ }^{27}\right)$.

- Ectasias aneurismáticas del cayado y/o de sus tributarias $\left(^{30-36}\right)$.

- Confluentes en "delta" $\left({ }^{12.14}\right)$.

- Ausencia de cayado. La VSE en la pierna o el muslo se divide en múltiples ramas arborescentes, cada una de las cuales alcanza el SVP a través de venas perforantes $(11.12,14$ ).

\section{MATERIALES Y MÉTODOS}

Se incluyó en un estudio retrospectivo todos los cayados de la VSE disecados en el transcur- 
A

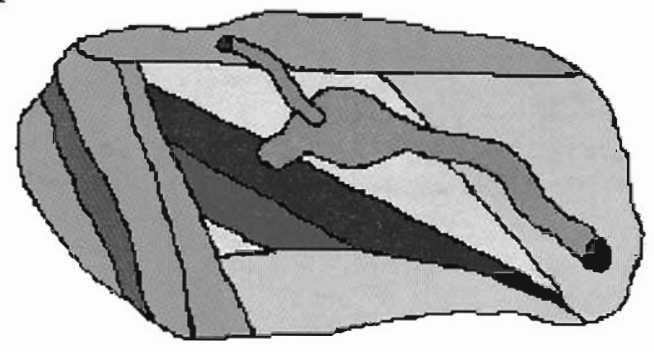

C

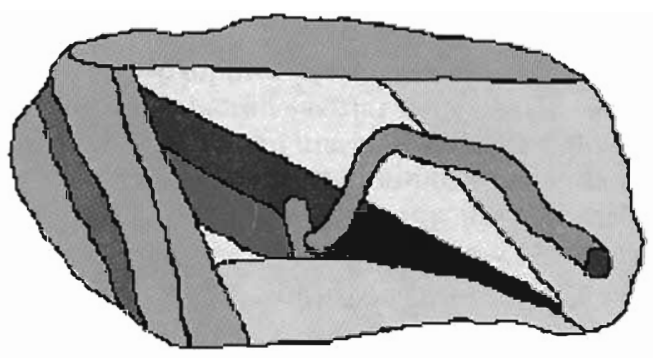

B

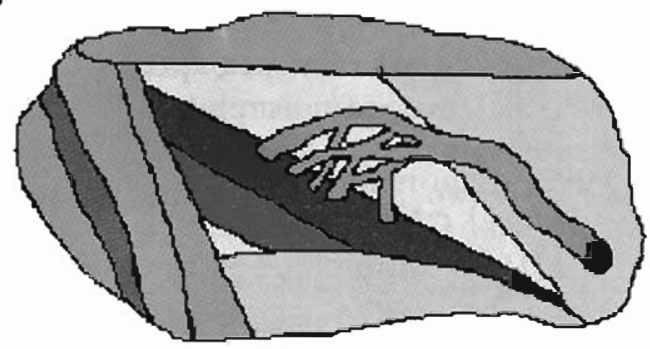

$\mathrm{D}$

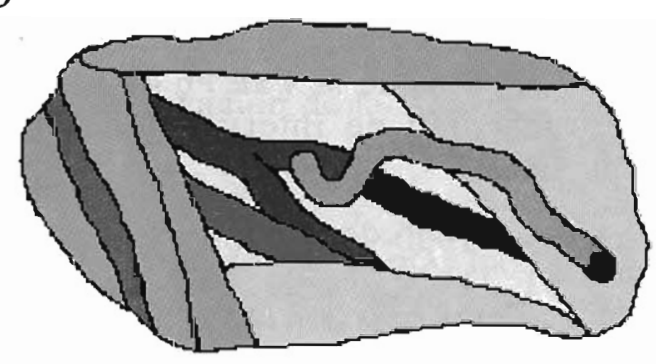

Figura 3.- Anomalías del contluente safenopoplíteo. A. Ectasia aneurismática del cayado de la VSE.

B. Terminación de la VSE en "delta de río". C. La VSE se vierte en una tributaria de la vena poplitea: vena del sóleo, venas gemelares, vena ue Dodd. D. Fusión alta (en el hueco poplíteo o superior) de ambas venas tibiales, originando una imagen de desdoblamiento subyacente de la vena poplítea. La VSE se vierte en una de las ramas.

so de intervenciones por várices esenciales en nuestro Servicio, entre los meses de encro de 1990 y encro de 2001.

En todos los pacientes se revisó las historias clínicas, registrando las pruebas preoperatorias practicadas y los hallazgos anatómicos rellcjados en la hoja operatoria.

Protocolo quirúrgico. A partir de 1996, se intentó constatar, en el transcurso de la intervención, los siguientes datos: altura y forma del confluente safenopoplíteo (Figura 2), vena en la que desembocaba la VSE y número de colaterales en el cayado; así como cualquier otra anomalía anatómica que pudicra apreciarse (Figura 3 ).

Para establecer la altura de la unión, tomamos las mismas referencias que Creton $\left({ }^{24}\right)$ :
- Unión normal: situada entre el pliegue poplíteo y los siguientes 7 centímetros en dirección craneal.

- Unión alta: localizada más allá de 7 centímetros cranealmente, tomando como referencia el pliegue poplíteo.

- Unión baja: situada por debajo del pliegue poplíteo.

Posteriormente, se procedió a la confección de la hoja operatoria, reflejando minuciosamente todos los hallazgos. La nomenclatura y criterios clasificatorios fueron adaptados a los propucstos en la literatura $(2.49 .11 .12 .14)$.

Pruebas preoperatorias. Hasta el año 1998, únicamente se practicó pruebas complementarias (Mebografía ascendente y/o EDC), ante la 
presencia de factores de ricsgo de trombosis venosa prolunda o cn casos scleccionados. Los pacientes provenientes de la lista de espera quirúrgica de la provincia de Hucsca aportaban habitualmente una ncbografia ascendente.

Desde 1998, disponemos de EDC en nuestro centro (Logiq $400 \mathrm{CL}^{*}$, Gencral Electric, con sonda de $7 \mathrm{MHz}$ ), solicitándose de rutina en lodos los casos.

\section{RESLLTADOS}

En el período de tiempo estudiado. se disecó un toral de 47 cayados de la VSE en 41 pacientes, en el transcurso de intervenciones por várices esenciales.

En el intervalo 1990-95, lueron 15 los cayados de la VSE (13 pacientes) intervenidos quirúrgicamente (Tabla 1). En un único pacientc se réalizó en la misma opcración cxéresis combinada de ambas venas safenas. La altura del cayado lue el dato más habitualmente rellejado ( $27 \%$ de los protocolos quirúrgicos), seguido de la vena en que vertía su flujo la VSE, expresalmente reseñada cn una quinta parte de los casos.

En cl intervalo 1996-01 sc disccó 32 cayados de la VSE (28 pacicntes). En 14 casos (44\%) sc llevó a cabo crosectomía y stripping de ambas salenas en el transcurso de la misma intervención quirúrgica. En cuanto a la altura de la descmbocadura saficniana, la mayor frecuencia correspondió al hueco poplíteo con 23 casos (72\%), por encima del hucco popliteo en $5(16 \%)$ c infrapoplítea en $2(6 \%)$. En $2(6 \%)$, su localización no aparecía reflejada correctamente en la hojia operatoria.

La VSE finalizó en la vena poplítéa en 20 casos $(63 \%)$, lo que la convirtió en la terminación más frecuente de ruestra scrie. La VSI lue la destinataria de la VSE en otros 4 casos, en 1 de cllos mediante una terminación del tipo $C$, en 2 del tipo E y en el otro del tipo F (Figura 2). En $56 \%$ de los casos se identificó colatcrales de una cicrta importancia en el cayado de la VSE.
Tabla 1.- Variantes analómicas del confluente safenopopliteo en 47 cayados disecados en pacientes inlervenidos por varices esenciales. Se delalla lus hallazgus según diferentes calegorias y divididos $\mathrm{cm}$ dos períndos de tiempo: 1990-95 y 1996-01

Scgún la altura del cayado de la VSE

$\begin{array}{cc}1990-95 & 1996-01 \\ - & 2(6 \%) \\ 3(20 \%) & 23(72 \%) \\ 1(7 \%) & 5(16 \%) \\ 11(73 \%) & 2(6 \%)\end{array}$

Según la vena en la que desemboca la VSE

$1990-95 \quad 1996-99$

$\checkmark S E$ en la poplitea

$2(13 \%) 20(63 \%)$

VSF en una rama de la poplitea - $3(9 \%)$

VSEen la VST $\quad-\quad 2(6 \%)$

Mixta (VSI y poplítea)

$2(6 \%)$

VSË en la fomoral profunda $\quad 1(7 \%)$

No determinado

$12(80 \%) 5(16 \%)$

Seguin el número de tributarias en el cayado $1990-95 \quad 1996-99$

$\begin{array}{lcc}\text { Ninguna } & - & 11(35 \%) \\ \text { Unad } & 2(13 \%) & 10(31 \%) \\ \text { Dos } & - & 7(22 \%) \\ \text { Mas de dos } & & 1(3 \%) \\ \text { Nodelerminado } & 13(87 \%) & 3(9 \%)\end{array}$

En cuanto al resto de anomalías del cayado, la más recuente fue la cctasia venosa en el hucco poplíteo (4 casos). En 2 ocasiones, cl diámetro era superior a 1,5 centímetros, por lo que lucron considerados auténticos ancurismas venosos. En un paciente se trataba de una vena del área poplítea (o de Thiery) arrosariada, y en orros 3 cra la propia VSE la que csiaba dilalada sacularmente.

En 3 ocasioncs, la VSE formaba un tronco común con la vena gemelar interna antes de la desembocadura en la poplítea (Figura 4).

En cuanto a las prucbas complementarias, en el intervalo 1990-1995, tres pacientes acu- 


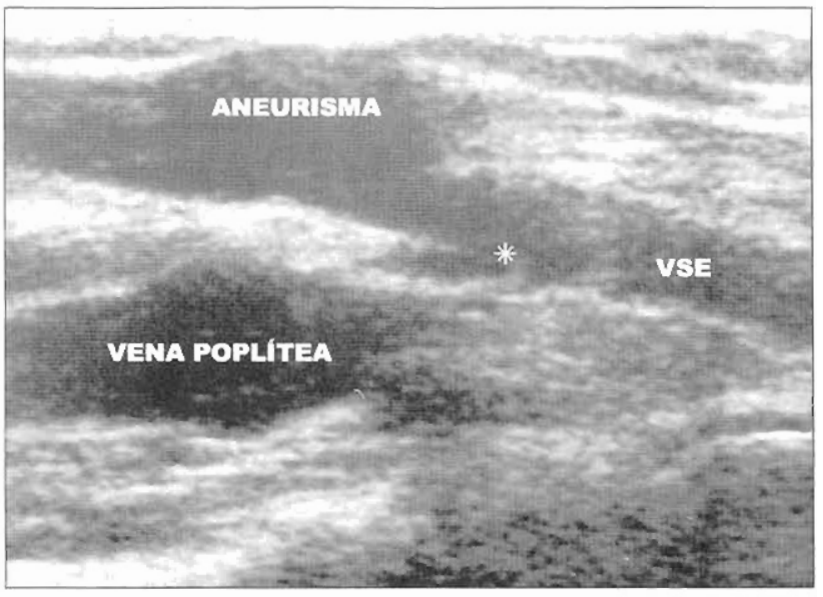

Figura 4.- Eco-Doppler preoperatorio en el que st muestra una dilatación sacular aneurismática del cayado de la VSE en una mujer de 35 añus. Se visualiza igualmente una vena gemelar desembocando en la VSÉ ('l).

dieron al quirólano cun una licbogralía aseendence $(20 \%)$.

En cl periodes 96-01, se deinandó prucbas de imagen col 19) de los 28 paciculcs $(68 \%), 5$ lichogralias asecudentes, I8 EDC y 2 varicugrallias.

De los 18 EDC practicados. Ia concordancia de) dagnóstico radiológico con los posteriores hallazgos en quiróanes (conkerdancia ceo-quirúrgica) se estableció en los siguicntes poreentajes.

1. Altura del conlluente VSE/SVP: $78 \%$ (1 4 de 18 accrtadas $)$

2. Vena destinalaria de la VSE: $33 \%$ (2 de las 6 terminaciones no poplícas con EDC lucron detectadas).

3. Ectasias venosas: $75 \%(3$ de 4$)$.

\section{DISCLSIÓN}

La incención con que se aborda prec e intraperatoriamente la biseceión del cayado de la VSE en nuestro Servicio, hat experimentado un cambio radical deste 1996 hasta hoy.
En una primera ctapa (90-95), la ausencia de prucbas properatorias era habitual y. cuando cran solicitadas, cral exclusivamente para descarlar problemas en el SVP. Por cllo, cl cartograliado preoperalorio se realizalhat en el antequirólano, mediante visión directa y maniohras de compresión. El objerivo básico de la disección del cayado de la VSE crie permitir el stripping de la velua, sin ancidir en aspectos. como una ligadura alta os en la posible incompoconcia de las venas gemelares, parat posteriornence tratar las perforantes insulicicntes y las tributarias dilatadas. Las hojas operatorias tan sólo rellejaban datos anatómicos ante la presencia de hallazgos incsperados y muy cvidentes (una cerminación de la VSE cn la femoral profuncla, una vena de Giacomini de gran diámetro. cintre otross.

Actualmente. las pautas bajo las que practicamos la cirugía en cl cerritorio de la VSE son:

- Desde 1998, eco-Dupler de rutina en codos los casos. solicirando al radiólogo una especial atención hacla la localización y las posibles variantes analomicas del conlluente salionopoplíco.

- Mericuloso cartograliado properatorio del cayaldo VSE. de las perlorantes insuficiontes y las trihutarias dilatadas, siguicndo las indiCaciones del EDC y nuestros propios hallazgos exploralorios.

- Una vezen el quirólano, colocación del paciente en una bucna posición, óptima ilumiración y un abordaje sobre el cayado de la VSE amplio y correcamence siluado, que nos permita un excelente campo quirúrgico. La localización de la incisión curánca puede ser individualizada en lunción de las pruebas preoperatorias, aunque habitualmente cmpleamos la vía horizoncal sobre el plicgue poplitco $\left({ }^{37}\right)$.

- La VSE cstá involucrada cotal o parcialmence en $90 \%$ de las recidivas en el cerritorio saleno incerno (25.38). Pur cllo, siemprevaloramos la necesidad de tratar la VSE cuando vamos a practicalr cirugía en el territorio de 
la VSI y se comprueba várices en la cara posterior de la pierna y/o insuficiencia del cayado de la VSE en el EDC. Ello ha motivado, muy probablemente, el aumento de las exéresis combinadas de ambas safenas en una misma intervención.

- Al igual que en la VSI, siempre asociamos una fleboextracción larga a la crosectomía de la VSE, salvo que exista alguna contraindicación $\left.{ }^{26,37.38}\right)$.

- Identificación, cuando ello es posible, de la misma unión safenopoplítea, buscando una ligadura alta del cayado $\left({ }^{37.39}\right)$. El motivo es prevenir el abandono in situ de un muñón residual largo, causante de hasta un $75 \%$ de las recidivas en el territorio safeno externo $\left({ }^{2.39-41}\right)$

- Identificación y tratamiento, si procede, de las venas gemelares, salvo que existan dificultades técnicas, ya que participan o son la causa fundamental de hasta $2 / 3$ de las recidivas $\left({ }^{5.26}\right)$.

- Búsqueda de cualquier otra anomalía que pudiera presentarse. Tratamiento del resto de perforantes y tributarias.

- Desde 1996, minuciosa confección de la hoja operatoria.

En cuanto al diagnóstico, y dada la complejidad del hueco poplíteo, tan sólo el EDC es capaz de determinar correctamente la anatomía venosa, fijar los puntos de reflujo $\left({ }^{3 .}\right)$ y resolver un posible diagnóstico difcrencial entre las dilataciones ampulares de la VSE, quistes de Barker, trombosis venosas profundas y los aneurismas de la arteria poplítea $\left({ }^{42}\right)$.

El EDC es una prueba con bajo costo económico y múltiples ventajas para el paciente: cs atraumático, indoloro, no invasivo, provoca nula irradiación, tiene una excelente tolerancia (lo que ofrece la posibilidad de repctir la prueba cuantas veces sc precise) y no modilica la hemodinámica venosa. Como único problema, se plantea la neccsidad de contar con un explorador que posea amplios conocimientos anatómicos, hemodinámicos y flebológicos $\left({ }^{3.43}\right)$.
La tasa de aciertos del EDC y, por tanto, su fiabilidad, puede ser medida adecuadamente confrontando la concordancia eco-quirúrgica. Si consultamos diversos reportes de la literatura, encontramos una tasa de $90 \%$, evaluando la altura del confluente $\left({ }^{8}\right)$, un $80 \%$ al establecer las relaciones entre la VSE y las venas gemelares $\left({ }^{3.7 .29}\right)$, y casi un $100 \%$ cuando se determina los puntos de reflujo $\left({ }^{29}\right)$. Por nuestra parte, los resultados que hemos obtenido con el EDC han sido satisfactorios, aunque distantes todavía de los que se presenta en otras series; a pesar de todo, pensamos que una práctica cada vez mayor, y la presencia simultánea del cirujano y el radiólogo durante la realización de la prueba, han incrementado significativamente su rendimiento diagnóstico.

La adopción del EDC como prueba de referencia en flebología ha supuesto el abandono casi total de la flebogralía ascendente y, por añadidura, la renuncia a llevar a cabo un stripping "a ciegas", sin una exhaustiva valoración hemodinámica de la VSE, de la válvula preostial y de sus tributarias y perforantes $\left({ }^{44}\right)$. Únicamente seguimos indicando de forma ocasional la llebografía selectiva (varicografía), y ello en dos sicuacioncs especiales: en las recidivas complejas poplíteas o ante la presencia de dilacacioncs aneurismáticas en el cayado de la VSE.

En nuestra experiencia, el hallazgo anatónico más estrcsante para el cirujano son las ectasias aneurismáticas de la VSE o sus ramas en el hueco poplíteo $\left({ }^{30-36}\right)$, ya que dificultan la identificación de estructuras y son propensas al sangrado por la debilidad parietal. Nuestras principales complicaciones han sido de índole menor $\left({ }^{45}\right)$, principalmente parestesias, en ocasiones dolorosas, y sensación de "acorchamiento" en el talón y el pie, probablemente originadas por la lesión del nervio safeno durante la fleboextracción. Los hematomas posteriores al stripping localizados en el trayecto de la VSE son particularmente molsstos para el paciente, dada la inextensibilidad de la celda aponeurótica que los alberga, y suelen precisar tratamiento mediante una incisión evacuadora. 
En cuanto a las recidivas en el territorio VSE, han sido evaluadas en $50 \%$ a los 5 años de la primera incervención ( $\left.{ }^{6}\right)$. La causa más trecuente $\left({ }^{40.41 .46}\right)$ es una cirugía previa deficienic, con abandono in silu de la unión safenopoplítea y un muñon safeno largo ( $38 \%$ a $87 \%$ de los casos, según la serie consultada). Otros motivos son las perforantes o venas gemelares no tratadas $(12,5 \%$ a $34 \%)$ o las neosafenas $(3,5 \%$ a $52 \%)$.

Hemos reintervenido 6 casos propios de recidivas mayores que precisaron revisión poplítea, aunque resulta casi imposible valorar su porcentaje en un hospital de primer nivel en el que se opera lista de espera ajena. Actualmentc, la Mebectomía segmentaria bajo anestesia local sin ingreso (asociada en ocasiones a la microcoagulación Parvulesco y la transfixión de Brinzeu) nos permite resolver satisfactoriamente la mayoría de las recidivas de escasa o mediana entidad $\left({ }^{47.48}\right)$.

Como conclusión, la protocolización de nuestras actuaciones diagnósticas y terapéuticas, la disponibilidad de EDC, la búsqueda de diagnósticos anatómicos intraoperatorios precisos y la confección de hojas operatorias detalladas han representado, en su conjunto, un avance sustancial en la calidad de la cirugía en el territorio de la VSE en nuestro Servicio.

Igualmentc, estamos convencidos que nos han ayudado en la consecución de los tres objetivos básicos e irrenunciables para cualquier cirujano que practique cirugía flebológica $\left({ }^{48}\right)$ : ausencia de complicaciones intra y posoperatorias, baja tasa de recidivas y obtención de un resultado estético, plenamente satisfactorio para el paciente.

\section{BIBLIOGRAFÍA}

1) Natali J. Conséquences médico-légales des complications de la chirurgie superficielle des membres inférieurs. Phlébologic 1993: 46(4):613-8.

2) Creton D. Écude anatomo-chirurgicale de 41 jonctions saphéno-poplitées incontinentes. Peut-on réduire le risque de récidive variqueuse poplitée? Phlébologie 1998: $51(4): 457-63$
3) Díaz Gónez F, Castells Ferrer P, Recio Valenzuela E, García Armengol J, Farnández Martínez C, Roig Vila JV. Escudio comparativo entre la flebografía poplitea dinámica y el Eco-Doppler en color en el diagnóstico de la insuficiencia venosa en el hueco poplíteo. Cir Esp 1998. 63(5):373-7

4) Payen B. Rappcl anatomique de la veine saphìne externe Phlébologie 1985; 38(3):453-61.

5) Gorny Ph, Payen B, Reinharez D, Hutinel B, Blanchemaison $\mathrm{Ph}$, Tran-Duy $\mathrm{S}$. Chinrgie de la saphène externe: Conclusions pratiques d'après uné étude sur 225 interventions. Phlébologie 1994: 47(4):383-8.

6) Franco G. Exploration ultrasonographique des récidives du creux poplitée après chirurgie. J Mal Vasc 1997: $22: 336-42$

7) De Sinione JG. Echo-doppler couleur de la crosse conmune petite saphène-veines jumelles. Aspects anatomiques et hémodinamiques. Phlébologie 1998; 51(2): 169-77.

8) Lemasle P, Uhl JF, Lefebvre-Vilardebo M, Tamisier D, Baud JM, Cornu-Thenard A. Confrontation échochirurgicale de la terminaison de la saphène externe dans le cadre de la chirurgie d'exérèse: Résultats préliminaires. Plhlébologie 1995: 48:321-7.

9) Mercier R, Fouques Ph, Portal N, Vanneuville G. Anatomic clirurgicale de la veine saphène externe. Consequences chirurgicales dans le trajtement radical des varices du mumbre inférieur. J Chir (Paris) 1967; 93(1):59-70.

10) Vasdekis SN, Clarke GH, Hobbs JT, Nicolaides AN. Evaluation of non-invasive and invasive methods in the assessment of short saphenous vein termination. Br J Surg 1989: 76(9):929-32

(1) Cibor Z, Cencora A. La veine saphène externc. Données anatomiques. Phlćbologic 1968: 21(3):257-65.

12) Thiery L. Anatomie chirurgicalc de la fosse poplitée. Phlébologie 1986; 39(1):57-66.

13) Schnyder P, Ramelet AA. Anatomia de las venas de las extremidades inferiores. En: Ramelet AA, Monti M, editores. Manual de Flebología. Barcelona: Masson S. A.; 1992. p. 3-19.

14) Blanchemaison Ph, Greney Ph, Camponovo J. Atlas de anatomia de las venas superficiales del niembro inferior. Volumen III: "El territorio de la safena externa. Las venas del pie". Madrid: Publicaciones Médicas de Laboratorios Servier; 1999. p. 6-49.

15) Corcos L, Peruzzi G, Romen V, Dini S. Papel de la safena externa en las varices primitivas y secundarias de los miemhros inferiores. Angiología 1985; 37:124-35.

16) Blomquist HE. Variability in the terminations of the short saphenous vein in the Fints. Ann Chir Gynaecol Fenn 1968; 57(1):55-8.

17) Perrin :M. Anatomie el physiologie normales et pathologiques des veines des membres inférieurs. Rev Prat (Paris) 1994; 44(6):717-25.

18) Furderer CR, Marescaux J, Pavis D'Escurac X, Stemmer $R$. Les crosscs saphéniennes. Anatomie et concepts thérapeutiques. Plhlébologic 1986: 39(1):3-14. 
19) Kosinski C. Observations on the superficial venous system of the lower extremity. J Anat 1926: 60:131-42.

20) Dodd H. The varicose tributaries of the poptiteal vein. Br J Surg 1965; 52(5):350-4.

21) Engel AF, Davies G, Keeman JN. Preoperative Incalisation of the sapheno-popliteal junction with duplex scanning. Eur J Vasc Surg 1991: 5:507-9.

22) Haeger $\mathbf{K}$. The surgical anatomy of the sapheno-femoral and the sapheno-popliteal junctions. J Cardiovasc Surg 1962; 3:420-5.

23) Moosnan DA, Hartwell SW. The surgical significance of the subfascial course of the lesser saphenous vein. Surg Gynecol Obsiet 1964; 115:761-6.

24) Creton D, Kohler F. L'incontinence de la petite veine saphène est-elle en relation avec la hauteur de son abouchement dans la voie profonde. Phlébologie 2000: 53(1):7-12.

25) Perrin M, Lepretre M, Becker F. La place de la saphène externe dans la chirurgie itérative des varices essentielles des membres inférieurs. Phlébologie 1980: 33:321-27.

26) Rettori R. Avenir des résections sysrematiques de la veine sap̧hène externe. Phlébologie 1982; 35(4):843-50.

27) Cadere T. La saphène externe. Anatomie et pathologie. Plalébologie 1980: 33(2):275-80.

28) Garcia Amiangol J, Castells Ferrer P, Recio Valenzuela E, Roig Vila JV, Fernández Martínez C, Calvete Chornet J, Díaz Gómez F, Buch Vila E, Lledó Móstoles S. Diagnóstico y tratamiento quirúrgico de las varices por insuficiencia venosa a nivel del buéco poplítco. Angiología 1994: 3:99-103.

29) Guillet JL, Perrin M, Hiltbrand B, Bayon .M, Gobin JP, Calvignac JL, Grossetête. Apnort đe l'Écho-Doppler pré et postopératoire dans la chirurgie vejneuse superficielle de la rosse poplitée. J Mal Vasc 1997; 22:330-5.

30) Thiery L. Les anomalies veineuses de la fosse poplitéc. Phlébologie 1988; 41(4):729-34.

31) Calligaro KD, Ahmad $S$, Dandora R, Dougherty MJ, Savarese RP, Doerr KJ, McAffee S, De Laurentis DA. Venous aneurysuns: Surgical indications and review of the literature. Surgery 1995; 117(1):1-6.

32) Gillespie DL, Villavicencio L, Gallagher C, Chang A, Hamelink JK, Fiala LA, el al. Presentation and management of venous ancurysms. J Vasc Surg 1997: $26(5): 845-.52$.

33) Kin DH, Lescault EJ. Aneurysm of the small saplaenous vein presenting as a popliteal mass. A case report. Am J Orthop 1999: 28:304.
34) Ramadan F, Johnson G. Primary lesser saphenous vein aneurysm in a child. J Pediatr Surg 1991:26:738.

35) Schatz IJ, Fine GF. Venous an'urysms. N Engl J Med $1962 ; 266(25): 1310-2$.

36) Uematsu M, Okada M. Primary venous aneurysms. Case reports. Angiology 1999: 50:239

37) Creton D. Aspecus techriques de l'éxerese de la petite veine saphène. Phlébologie 1999: 52(2): 169-74

38) Megret G. Étude comparative entre l'examen clinique et l'examen doppler acouscique de la saphènte externe. A propos de 191 cas. Phlćbologie 1985:38(3):469-73.

39) Vandendriessche $M$. Récidive dans la creux poplitée. Phlébologie 1986: 39(1):67-7!

40) Creton D. Récidive variqueuse poplitée après chinrgie du reflux saphène externe. 56 réintervencions poplitées. Phlébologie 1996: 49(2):205-12.

41) Elbaz C. Chirurgie itératjve dans les récidives après opération pour varices essentielles. Phlébologie 1977: 30(3):265-73

42) Tournay R. Erreurs de diagnostic possibles en phlćbologie concernant des affections du creux poplitéc. Phlébologie $1965 ; 18(3): 235-8$

43) Mercer KG, Scott D.JA, Berridge DC. Preoperative duplex imaging is required hefore all operations for primary varicose veins. Br J Surg 1998: 85:1495-7.

44) Pieri A, Vannuzzi A, Duranti A, Michelagnoli S, Marcelli F, Santini M, et al. La valvule pré-ostiales de la veine saphène externe. Varices atypiques jambières dues à l'incontinence (fonctionnelle) de la jonction saphénopoplitée. Écude par écho-Duppler couleur. Phlébologie 1997: 50:343-50.

45) Rettori R. Accidents du traitement chirurgical des varices. Phlébologie 1982:35(1):391-2.

46) Daniel C, Lemasle P. Apport de l'écho-Doppler couleur dans la récidivé post-cbirurgicale de la petite saphéne. Revué de la Littéranre. Phlébologie 1998; 51(2): 179-88.

47) Sánchez Beorlegui J, García Calleja JL, Fernández Ruiz M, Lamata Hernández F, Gangutia Hernández S, Córdoba Diaz De Laspra E. Flebectomía segmentaria ambulatoria (FSA). Técnica y resultados preliminares en 28 pacientes. Cirugía Mayor Ambulatoria 1999; $4(1): 342-3$.

48) Santos Gastón MA. Microvarices. Una asignatura pendiente de la flebología estética. Nlfaro (España): Grăficas García: 1996. p. 154 